\title{
Pédagogie universitaire et partenariat université- entreprise : enjeux, écueils, perspectives
}

Loïc Brémaud et Michel Boisclair

\section{OpenEdition}

Édition électronique

URL : http://journals.openedition.org/ripes/577

DOI : 10.4000/ripes.577

ISSN : 2076-8427

Éditeur

Association internationale de pédagogie universitaire

Référence électronique

Loïc Brémaud et Michel Boisclair, « Pédagogie universitaire et partenariat université-entreprise : enjeux, écueils, perspectives », Revue internationale de pédagogie de l'enseignement supérieur [En ligne], 28(1) | 2012, mis en ligne le 20 avril 2012, consulté le 08 septembre 2020. URL : http:// journals.openedition.org/ripes/577 ; DOI : https://doi.org/10.4000/ripes.577

Ce document a été généré automatiquement le 8 septembre 2020

Article L.111-1 du Code de la propriété intellectuelle. 


\title{
Pédagogie universitaire et partenariat université-entreprise : enjeux, écueils, perspectives
}

\author{
Loïc Brémaud et Michel Boisclair
}

\section{Introduction}

1 Penser la relation entre pédagogie universitaire et partenariat université-entreprise suppose d'explorer les interactions entre deux mondes: d'une part, le monde de la recherche et le monde de la production de savoir et, d'autre part, le monde social et économique de production de biens et de services, mondes qui, pendant longtemps, se sont ignorés et ont évolué de manière autonome. Ce numéro thématique de RIPES se propose d'étudier les facteurs qui ont imposé ou permis ce rapprochement, les formes produites par l'alchimie de la rencontre de ces deux pôles. Enfin, il s'agira de relever les conditions favorables à l'émergence de dispositifs de formation universitaire à caractère professionnalisant nés de cette rencontre, qu'ils soient diplômants ou débouchant sur des formations continues d'adultes, créditées ou non.

2 Si la question de la professionnalisation dans le cadre des formations diplômantes ou certifiantes (Bourdoncle, 2000 ; Wittorski, 2008) est aujourd'hui largement débattue au sein des institutions universitaires de tous pays, quelle place conviendrait-il de laisser aux entreprises prises dans leur acception large appartenant au secteur privé, public ou parapublic? Comment penser cette relation dans la complémentarité ? Comment imaginer les ponts (Malglaive \& Weber, 1983) entre les mondes d'émergence des savoirs pratiques et d'action (Barbier \& Galatanu, 2004), d'une part, et les lieux de production de la recherche et des savoirs théoriques, d'autre part? Comment repenser la complexité de ces dispositifs en intégrant aspects formels, non formels et informels, pour s'ouvrir à de nouvelles configurations (Brémaud \& Guillaumin, 2010) ?

3 Ce numéro thématique de RIPES s'exercera donc à explorer plusieurs dimensions de la production en processus de cette relation, comme pensée en archipel (Deleuze, 1989) 
" comme l'affirmation d'un monde en processus. Non pas même un puzzle, dont les pièces en s'adaptant reconstitueraient un tout, mais plutôt comme un mur de pierres libres, non cimentées, où chaque élément vaut pour lui-même et pourtant par rapport aux autres » (p. 192). Ainsi, il s'agira de répondre à une multiplicité de questions posées en matière d'ingénierie pédagogique à l'institution universitaire, engendrée par la mise en relation de ces deux pôles, autour du triptyque professionnalisation-partenariatalternance :

\footnotetext{
- Comment définir la professionnalisation dans des cadres socio-économiques, mais aussi institutionnels aussi différents que ceux rassemblés autour de la francophonie? Quelle ingénierie pédagogique et de formation concevoir pour garantir l'établissement de véritables dispositifs de formation, au sens large (formations modularisées, conseil en gestion, accompagnement individualisé ou d'équipe et collectif, mentorat...), susceptibles d'être produits dans le cadre d'une association entre universités et entreprises? Quelle place donner aux différentes entités de l'université dans ce cadre pour que les dispositifs soient véritablement professionnalisants : rôle de la direction, des responsables de formation universitaire, place des services assurant des prestations de formation continue pour les entreprises?

- Comment instaurer un véritable partenariat, permettant à chacun de conserver ses spécificités et d'établir des relations productives et durables ? Comment créer des relations d'interdépendance permettant d'éviter toute « fusion-confusion », et de dériver vers une simple relation client-fournisseur?

- Ce partenariat doit-il ou non reposer sur un système d'alternance pour permettre la professionnalisation? Si oui, comment créer un tel dispositif permettant de reconnaître deux lieux de formation et d'apprentissage (Geay, 2007) ? Comment en tant qu'ingénieur pédagogique combiner à la fois du " génie » c'est-à-dire s'appuyer sur un corpus de connaissances identifié et maîtrisé, de «l'ingenium » des aptitudes cognitives de reliance, et de « l'ingenior » à savoir les capacités créatives de tout ingénieur (Brémaud, 2010) ?
}

4 Avant de permettre aux auteurs de la revue de répondre à toutes ces questions, pouvons-nous éclairer les processus qui ont progressivement permis d'encourager ce rapprochement entre universités et entreprises, ouvrant sur de nouvelles modalités d'ingénierie pédagogique, à partir de l'établissement progressif du triptyque "professionnalisation-partenariat-alternance»? Il nous est nécessaire de nous replonger dans l'histoire des universités et de ses relations avec les pouvoirs politicoéconomiques des différentes époques. Nous nous appuierons, à cet effet, largement sur l'ouvrage de Christophe Charle et de Jacques Verger (1994) sur l'histoire de l'université.

\section{L'université et le pouvoir socio-économique : des rapports de force qui évoluent selon les contextes}

\subsection{Des temps fondateurs à l'étatisation progressive de l'université}

5 Au sein du monde occidental, les universités émergent vers le début du XIIIe siècle et sortent ainsi délibérément de l'étroit cadre diocésain qui était celui des écoles antérieures. Charle et Verger (1994) détaillent ainsi comment maîtres et étudiants se réunissent pour se donner des statuts, créer les conditions d'une entraide mutuelle, garantir leur protection face aux menaces possibles de la population et des autorités locales et règlementer l'exercice autonome de l'activité qui était la raison même de leur 
association, à savoir l'étude et l'enseignement. Ces maîtres laïcs transgressent l'ancienne doctrine de Saint Bernard selon laquelle on ne peut enseigner la science qui n'appartient qu'à Dieu. Ainsi, en obtenant cette autorisation, ils s'émancipent de leur position subalterne, à la manière des bourgeois des villes et artisans de métiers. Finalement, l'Université va leur accorder, à Paris, au début du XIIIe siècle, tous les signes de la corporation: un sceau, le droit de grève, des organes de direction, des statuts, des programmes et des dirigeants appelés « régents » (Dubar \& Tripier, 1998, p. 26). L'université devient ainsi une corporation et le maître en est la figure éminente. Sous la pression de l'étatisation progressive des sociétés européennes et sous des formes diverses, le contrôle politique s'appesantit sur les universités. En échange de cette intégration à l'ordre commun du royaume, les universitaires obtinrent à la fois certaines gratifications financières ainsi que des promesses de belles carrières. « Ils se plièrent sans peine en échange de faveurs du Prince, au rôle assigné : dispenser un savoir orthodoxe, former les futures élites locales, contribuer à l'ordre social et politique établi » (Charle \& Verger, 1994, p. 19). Mais à partir du XVIIe et XVIIIe siècle, un décalage se creuse peu à peu entre l'enseignement universitaire et les courants novateurs des sociétés européennes, et compromet le pouvoir d'attraction de l'université. «On voit par exemple qu'un peu partout la noblesse tendait à déserter l'université et se doter d'un réseau propre d'établissements d'enseignement où ses fils pouvaient s'initier aux manières mondaines et aux disciplines indispensables aux gentilshommes (danse, équitation, langues étrangères)» (Charle \& Verger, 1994, p. 50). Ainsi recherche et innovation trouvaient des échos plus favorables pour les élites des sociétés en voie de modernisation "Si nombre d'auteurs et de penseurs importants de l'époque moderne sont passés par le collège et l'université, comme étudiants et parfois même comme professeurs, c'est généralement hors de l'université qu'ils ont élaboré leurs œuvres majeures ou fait leurs découvertes les plus significatives. À l'époque moderne, les académies et les sociétés savantes, les cours et les chancelleries, les salons, sans parler de la bibliothèque ou du cabinet du riche amateur, sont devenus des lieux les plus communs, non seulement de la sociabilité savante, mais de la recherche et de l'innovation » (Charle \& Verger, 1994, p. 53). Le modèle français des Grandes Écoles, unique en Europe, émergera au sein d'un pays à grande culture centralisatrice, qui se méfie de tout corporatisme et qui cherche avant tout le contrôle social étroit sur ses élites. On reprochait ainsi aux universités d'avoir perpétué, jusqu'en plein XVIIe siècle, des enseignements dépassés, reposant sur les vieilles autorités médiévales (Aristote, Hippocrate, Gallien...) et d'avoir a contrario ignoré ou refusé, par aveuglement corporatiste, tous les courants novateurs nés en dehors d'elle. Mais les évolutions qui s'exprimeront avec des nuances notables entre pays catholiques, hostiles aux Lumières, favorisant une certaine sclérose intellectuelle, et ceux des pays protestants où diverses initiatives pédagogiques, comme l'abandon du latin, et la création de nouvelles chaires permirent de limiter le divorce entre universités et culture savante. Si l'université continue de former une partie des classes supérieures des sociétés en voie de modernisation et d'étatisation appartenant aux professions (médecins, avocats, notaires ...), sa perte d'influence auprès du corps social et notamment ses élites est certaine.

6 C'est Alexander Von Humboldt (1769-1859), naturaliste, géographe et explorateur allemand, qui jettera les bases d'un renouveau de l'université et va doter l'Allemagne d'un outil d'éducation adapté aux transformations de l'époque. Soubirou, en 2004, à travers un exposé de cours à l'institut d'études politiques de Paris défend qu'Humboldt, 
à l'opposé des penseurs européens d'alors, tient à lier fortement la nouvelle université à l'État, afin de lui permettre de maintenir ses objectifs élevés. Pour Soubirou, l'œuvre de Humboldt en matière de rénovation de l'université se résume en trois principes. Tout d'abord l'unité de la recherche et de l'enseignement, afin de disposer dans ce cadre des meilleurs savants, de les encourager à poursuivre leurs recherches et à les rendre collectivement responsables de l'attribution des diplômes et du niveau exigé. Le second principe est celui de la protection de la liberté universitaire, revendication traditionnelle des universités depuis le moyen-âge, qui comprend à la fois la Lehrgreiheit ou liberté d'enseigner et la Lernfreiheit ou la liberté de choix de cursus pour les étudiants. Enfin, le dernier principe repose sur l'abolition de la distinction entre "haute faculté », spécialisée et professionnalisante, préparant aux professions de théologie, de droit et de médecine et accessible seulement après une maitrise, et la «basse faculté " généraliste en arts et sciences. Ainsi, l'université Humboldtienne, tout en n'établissant pas de distinction entre ceux qui se vouent à la recherche et à l'enseignement, et ceux qui veulent étudier en vue d'entrer dans le monde du travail, suit-elle les évolutions à la fois sociale et économique des sociétés modernes. C'est ce que Macclelland (1980, cité dans Charle \& Verger, 1994) nomme la "professionnalisation» des universités allemandes. Système d'abord raillé par les universitaires français, ce modèle sera reconnu à la suite de la défaite de 1870 et servira de base à la rénovation des universités européennes des XIXe et XXe siècles, dans les temps forts de sa structuration en disciplines ${ }^{1}$.

\subsection{La post-modernité et les nouvelles missions de l'université}

Un double mouvement questionnera à nouveau le rôle et la place des universités dès le milieu des années soixante du siècle dernier : la massification des effectifs étudiants et l'entrée dans la post-modernité. Hier cantonnée, à côté des sociétés savantes, à faire émerger de nouvelles connaissances et à former les élites, l'université est confrontée à de nouveaux défis. Il ne s'agit plus de doter les étudiants d'une culture générale solide, d'une manière rigoureuse de raisonner, d'un art d'analyser minutieusement des textes et aussi des notions générales, de les doter des éléments d'une vision cohérente du monde, produisant la figure sociale de "l'intellectuel» selon Le Goff (1985, cité dans Charle \&Verger, 1994). Il s'agit d'abord et avant tout de préparer des classes d'âge nombreuses nées au cœur des trente glorieuses à un avenir professionnel, en raison d'une demande sociale forte d'élévation des niveaux d'éducation et de formation pour faire face au développement économique des sociétés industrielles occidentales (Bernier, 2011). Cette entrée dans la massification liée à l'émergence des classes moyennes sera accompagnée de la multiplication du nombre de diplômes, que ce soit en termes de niveau de qualification, qu'à l'intérieur des disciplines qui se sont multipliées depuis un siècle. Si, dans un premier temps, cette évolution rapide permettra d'assurer cette transition vers la professionnalisation par spécialisation plus poussée des formations universitaires, la tendance vers un découplage du marché des diplômes et celui de l'emploi se fera jour, par la montée du chômage des diplômés à partir des années quatre-vingt du siècle dernier. Les entreprises privées et publiques au sens large, accroissent leurs exigences en faisant entrer dans l'évaluation des salariés des capacités d'adaptation aux changements, d'initiative, d'autonomie, de communication, d'anticipation, et, plus généralement, des aptitudes non seulement physiques et cognitives mais également comportementales (Bourgault, Charih, Maltais

Revue internationale de pédagogie de l'enseignement supérieur, 28(1) | 2012 
\& Rouillard, 2003). Ce mouvement est largement autorisé par un changement de perception sociétale du monde de l'entreprise, un mouvement encouragé par certains auteurs comme Doray et Maroy (1995), l'heure du rapprochement entre éducation et économie semblant bien engagée. Ce rapprochement est décrié par Le Goff (1993) «Après avoir été largement considérée comme lieu d'exploitation et d'aliénation, l'entreprise s'est trouvée valorisée dans les années quatre-vingt comme elle ne l'avait jamais été auparavant. Bien plus : la logique productive et marchande, les méthodes managériales, l'esprit d'entreprise ont été érigés en modèle de référence. Ils constituent désormais des critères déterminants de la valorisation sociale du nombre d'activités et de l'aide que l'Etat entend leur accorder.» (p. 7). Les salariés doivent ainsi faire preuve d'initiative, de créativité et d'autonomie dans la décision individuelle et collective (Jobert, 1999). La post-modernité, qui s'installe en fin de siècle sous la poussée croissante de la mondialisation, met à mal les États-Nations auprès desquels s'étaient structurées les nouvelles universités du XXe siècle. L'entrée dans la société informationnelle (Boutinet, 1999) bouscule les temps et rythmes de production des connaissances. La rapide obsolescence des savoirs, le renouvellement permanent des réseaux informationnels conduit l'étudiant et le salarié à une position de perpétuel apprenant soucieux de digérer les changements incessants qui lui sont présentés. Il faut donc sans cesse retraiter de manière permanente ses repères cognitifs (Boutinet, 1999). La culture postmoderne s'impose ainsi sur une base volontariste à un niveau jamais atteint par aucune autre. Il est ainsi demandé à chacun, étudiant, salarié en formation permanente d'avoir à se justifier à travers une multiplicité de projets individualisants, émergents, structurants ... Niveau d'exigence qui s'applique d'autant plus aux nouvelles catégories de salariés issus de l'économie mondialisée et hyper-flexible, à savoir les intérimaires et intermittents (Voyer, 2011). Cette culture impose de se projeter, de s'insérer, de s'orienter, de se former (Boutinet, 1999). D'où la prospérité récente de la notion d'employabilité. Désignant à l'origine la probabilité de sortir du chômage, elle tend désormais à s'appliquer à l'ensemble de la population active et à devenir constitutive de la condition salariale contemporaine. Dans le contexte d'ouverture de la mondialisation de flexibilité productive des entreprises, les mobilités promotionnelle, fonctionnelle, professionnelle ou d'emploi deviennent la norme (Parlier, 2009).

8 A l'issue de la présentation rapide de l'évolution du rôle et de la place des universités dans les sociétés médiévales, étatiques puis espaces en voie de mondialisation, l'injonction faite à ces institutions est de plus en plus forte aujourd'hui afin qu'elles justifient de leur utilité sociale, que ce soit dans le domaine de la recherche ou de la formation (Lapointe, 2011). Ce phénomène est bien repérable par la montée en puissance de l'évaluation sous toutes ses formes ces dernières décennies. Il est également perceptible par l'émergence des classifications nationales et internationales des universités, censées guider le futur étudiant dans ses choix ou justifier du montant de la dépense d'investissement de ces lieux de formation et de recherche auprès des politiques. Les universités sont prises dans le marché ouvert de cette nouvelle demande sociale. Emportées dans le maelström des changements rapides de l'espace-monde, peuvent-elles y répondre seules pour accompagner ce rapide passage de la qualification à la compétence apparu à la fin du siècle dernier ? (Dadoy, 1990 ; Zarifian, 1988). 


\section{La professionnalisation : un nouveau paradigme de formation en pédagogie universitaire}

\subsection{Pédagogie universitaire et mutations du travail : pourquoi et comment professionnaliser aujourd'hui ?}

$\mathrm{Si}$, pendant plusieurs siècles, il s'agissait d'élaborer et d'enseigner le "savoir de l'honnête homme", les évolutions sociétales précisées plus haut ont modifié les missions de l'université au sein des pays développés, soumis à la concurrence mondiale (Freitag, 1998). Le travail et son évolution rapide se sont invités et imposent de nouvelles relations à cette institution. L'exemple du Canada illustre bien cette injonction nouvelle (Grosjean, 2005). Dans les années soixante, on pensait que l'expansion de l'enseignement supérieur contribuerait non seulement à la croissance économique de façon substantielle, mais aussi réduirait les inégalités en en rendant l'accès plus facile (Grosjean, 2005). Au cours des années soixante dix, l'enseignement supérieur produisait de plus en plus de diplômés, mais ces derniers n'avaient pas les qualités attendues par les employeurs. Dans les années quatre-vingt, le débat s'élargit et porta également sur les différents rôles de l'enseignement supérieur et la structure des parcours professionnels des diplômés (Squires, 1987). On tient davantage compte du domaine étudié, du programme proposé et de la demande des étudiants, ce qui mène à une multiplication des options et des stratégies individuelles. Dans les années quatrevingt-dix, un certain nombre de questions étaient à l'ordre du jour. D'une part, le regain d'intérêt pour l'employabilité des diplômés donna aux institutions d'enseignement supérieur la responsabilité de préparer les étudiants pour le monde du travail (Grosjean, 2005), en leur suggérant comment les étudiants devaient se préparer individuellement pour réussir professionnellement. D'autre part, on se demande si l'université doit offrir des qualifications « sur commande » au marché du travail. Or ces qualifications et cette employabilité peuvent-elles être produites seules par l'université, y compris dans le cadre des formations professionnalisantes? Mission impossible selon Jobert (1999) pour qui les méthodes traditionnelles de formation ne peuvent répondre aux injonctions nouvelles auxquelles sont soumis les salariés, comme être motivé, s'impliquer, prendre des responsabilités, prévoir, innover, s'adapter, communiquer, coopérer. Autant d'activités qui, selon lui, correspondent à un changement assez radical de la définition des savoirs pertinents et de leur acquisition dans l'action, que l'institution de formation seule ne peut produire (Dietrich, Gilbert \& Pigeyre, 2010; Labruffe, 2005). Cette intelligence qui se mêle à celle des consignes, des équipements et de la connaissance spécialisée, c'est celle des hommes et des femmes confrontés à la résistance des objets ou des situations sur lesquelles ils interviennent. Ainsi on parle de plus en plus de situation professionnelle, de situation de professionnalisation, de situation professionnalisante (Martin, 2006).

\subsection{Professionnaliser au sein de l'université : un changement de posture et de démarches de l'enseignant}

10 La pédagogie universitaire est ainsi confrontée à l'élargissement de ses perspectives, en redécouvrant les acquis, hier marginalisés en son sein, de la formation des adultes, en invitant de manière plus centrale le concept d'expérience. Mais contribution qui tient 
également à la clarification de ce concept dans le contexte de formation pour assurer une professionnalisation effective en tissant des liens avec deux courants théoriques (Debon, 2006) :

- Le lienavec l'apprentissage où les apports des théories de l'apprentissage constructiviste et socioconstructiviste sont dominants. Il faut noter que, bien avant le développement de la formation des adultes, ces théories ont renouvelé le développement des méthodes actives à l'école et le mouvement de l'Education Nouvelle avec Montessori, Freinet, Decroly, Cousinet pour ne citer que les pédagogues les plus connus, présents pour certains depuis le début du XXe siècle ;

- Le lien fait avec la compétence, concept sur lequel les approches développées en ingénierie de formation donnent un éclairage central (Dietrich, Gilbert \& Pigeyre, 2010 ; Foucher, 2010 ; Labruffe, 2005 ; Le Boterf, 2001, 2008).

11 Ainsi, contrairement à la position «traditionnelle et transmissive » de formation, il s'agit de développer une diversité de démarches et de moyens susceptibles de bien distinguer l'apprenant de l'élève, cherchant explicitement à rapprocher théorie et pratique. Il s'agit également pour le formateur de changer de posture. L'éducation ne résiderait donc pas dans l'acquisition d'une science pure et isolée pour en rechercher ensuite l'application à notre vie de tous les jours (Deledalle, 1995). Pour Barbier (2008), porteur de ce courant, enjeux sociaux, enjeux professionnels, enjeux personnels, enjeux de formation et enjeux scientifiques peuvent se révéler solidaires. L'organisation traditionnelle de l'enseignement supérieur, sur des bases disciplinaires classiques, privilégie la connaissance $d u$ fonctionnement $d u$ monde, opposant la formation professionnelle à la recherche. Les nouvelles organisations d'enseignement supérieur lient les champs professionnels et scientifiques, et privilégient la connaissance des processus de transformation du monde favorisant, ce faisant, l'articulation entre recherche et professionnalisation. Ce nouveau modèle privilégie souvent une référence épistémologique, théorique, méthodologique et sociale à l'activité humaine, référence transversale aux différents "champs de recherche/de pratique/de formation" concernés. Pour Clot (1995), l'étude du travail exige une fertilisation croisée permanente entre une clinique de l'activité, co-produite avec les milieux de travail et une démarche rigoureuse de conceptualisation appuyée sur des savoirs disciplinaires. Ce régime particulier de production des connaissances professionnalisantes consiste en « l'art de ménager » des passages entre rationalité de l'action et rationalité scientifique. Cette position suppose de revisiter les représentations nées des vieilles lignes de partage issues des anciens mondes sociaux, comme celui séparant les occupations des professions (Dubar \& Tripier, 1998), séparant les métiers manuels et les professions intellectuelles. Pour Bézille et Courtois (2006), la mise en avant du potentiel formateur de l'expérience vécue semble participer à un mouvement plus global de reconnaissance de la diversité des formes de savoir, profanes, savants, expérientiels, professionnels, existentiels, d'action... Mais aussi de leur légitimité respective et de leur complémentarité, au delà de l'opposition classique savoirs théoriques/savoirs pratiques.

12 Ces différents points de vue seront illustrés dans la première partie par trois contributions. Les auteurs débattront des questions posées, aujourd'hui, par la professionnalisation au sein des dispositifs de formation universitaire pris dans des contextes nationaux différents. 
13 Richard Wittorski, dans un premier temps, repositionnera «l'objet professionnalisation» "apparu dans un contexte du marché libre» de la postmodernité, au sein des nouvelles organisations professionnelles flexibles. Il précisera les différents sens de cette notion polysémique. Dans un deuxième temps, il mettra en perspective les leviers utilisés par les formations universitaires professionnalisantes de ces trente dernières années en France: dispositifs particuliers de la formation des enseignants et travailleurs sociaux, la place de la recherche-action professionnelle, l'introduction de la validation des acquis de l'expérience au sein des universités et son impact en matière de professionnalisation, la place des savoirs d'action ou pratiques et l'importance à leur donner dans les processus à visée de professionnalisation. Il clôturera ses développements en évoquant les conditions qui lui semblent devoir être réunies pour mettre en œuvre une formation professionnelle et/ou professionnalisante, en s'appuyant sur la combinaison des «six voies de la professionnalisation ».

14 Jérôme Eneau, Eric Bertrand et Geneviève Lameul, quant à eux, illustreront comment ces voies de professionnalisation peuvent s'appliquer dans le cadre d'une expérience menée dans une université française formant des ingénieurs. À partir de la perspective transformatrice de Mezirow, ils exposeront comment professionnaliser, aujourd'hui, des apprenants dans la "société de l'incertitude», dans un dispositif universitaire favorisant les dimensions auto-formatrices, co-formatrices et transformatrices. Il s'agit pour ces auteurs de combiner savoirs théoriques et savoirs pratiques dans un but d'émancipation des apprenants. Dispositif de formation constitué de plusieurs étapes et visant distanciation, recul réflexif et transformation des schèmes de pensée. Ils illustrent comment créer de «l'ingenium » et de «l'ingenior » dans un contexte "d'autoformation transformatrice", accompagnant des sujets autonomes se produisant (Mezirow, 2001). Ils présenteront la place particulière faite à la recherche dans ce dispositif de formation, imbriquée à deux autres temps pour produire une formation-action-recherche comme objet central structurant de l'espace dialogique de professionnalisation.

15 Enfin, pour clore cette première partie, Philippe Maubant et Lucie Roger développeront tout l'intérêt que les sciences de l'éducation doivent porter à cette notion. Ils lui donnent un relief particulier en la confrontant à d'autres concepts, comme celle de professionnalité, de savoirs professionnels. Ils replacent le développement des dispositifs de professionnalisation dans une perspective sociohistorique au sein de laquelle les corporations et groupes professionnels ont disposé d'un rôle déterminant. Ils illustreront leurs propos en s'appuyant sur les métiers supposant de fortes interactions humaines dans le cadre de relations de service et susceptibles de produire les activités suivantes: enseigner, former, soigner, accompagner, aider, assister, animer... Enfin, et dans le contexte particulier des universités québécoises, ils délivrent leur regard critique sur la professionnalisation des enseignants québécois, induite par la réforme de 2001 visant à inscrire la formation des maîtres dans une perspective de professionnalisation s'articulant autour de douze compétences professionnelles. Comme Richard Wittorski dans le contexte français, ils défendent pour le système québécois de formation des maîtres une position appelant à la mise en œuvre d'une dimension véritablement dialogique de la formation professionnelle en organisant le dialogue entre différents espaces-temps, permettant 
pour chaque apprenant la mise en récit de soi visant à interpréter ses expériences de travail, donc de donner sens aux différentes situations professionnelles rencontrées.

\section{Professionnaliser seul ou s'associer à des partenaires?}

\subsection{Le partenariat université-entreprise : une démarche incontournable}

16 L'histoire multiséculaire de l'université nous a permis de percevoir l'enchaînement successif des étapes qui l'ont distingué ou lié à la société, mais aussi de saisir les pressions et injonctions des différents pouvoirs politiques, économiques avec qui elle a dû composer. Si la professionnalisation suppose de repenser la pédagogie universitaire en encourageant des combinaisons de savoirs, bien au delà des savoirs disciplinaires sur leurs versants théoriques, comment concevoir les espaces permettant d'expérimenter les savoirs d'action et les savoirs pratiques décrits précédemment? Peut-on penser seuls les dispositifs professionnalisants, c'est-à-dire à partir des seuls dispositifs universitaires? Doit-on s'associer à d'autres pour donner corps à deux lieux d'apprentissage pour deux types d'apprentissage? Quels bénéfices tirer de ces partenariats? Quelles formes donner à ces dispositifs? A contrario, quels risques sont associés à ces assemblages. Ne risque-t-on pas d'y perdre son âme et pervertir les restes de l'identité d'une communauté constituée, dès sa naissance, d'hommes libres et critiques? En effet, tout pousse, aujourd'hui, aux rapprochements entre universités et entreprises. L'hypercomplexité d'un monde (Demorgon, 2008) qui ne peut plus se penser centralement a engendré la prospérité du terme gouvernance, comme processus de coordination d'acteurs, de groupes sociaux et d'institutions, en vue d'atteindre des objectifs définis et discutés collectivement (Le Gales, 2004). La notion de partenariat elle-même est d'usage très récent dans le domaine de l'éducation, comme le précise Zay (1994) « Partenariat est un terme récent. Il n'entre dans le Petit Larousse illustré qu'en 1987, contrairement à partenaire, dont l'origine remonte au XXVIIIe siècle, de l'anglais partner, lui-même altération, sous l'influence de part, de parcener ou parçuner, de l'ancien français parçonier, propriétaire indivis, co-partageant, de parçon, partage, butin, du latin partitio, partitionis (partage, séparation, division). Etymologiquement sont donc présents dans le terme à la fois l'avec et le contre, l'association et la division» (p. 719). Selon elle, l'évolution de la langue traduit celle d'un fait de société, dans le contexte particulier du système d'éducation français. Rien n'est plus étranger à l'école laïque de la Troisième République naissante que l'idée de partenariat. Elle se fonde dans la lutte pour arracher les enfants au monde du travail et enlever à l'Église le monopole de l'éducation des âmes. Elle dresse de hauts murs pour protéger ses élèves des vices de la rue. Parallèlement au déclin du marxisme, et plus nettement à partir des années quatre-vingt, l'idée de participation, le modèle libéral allemand pénètrent et se diffusent à partir des milieux d'entreprise, dans un climat de crise économique qui rend plus attractif l'emploi. Le partenariat en éducation serait donc lié à l'émergence du local, à l'importance croissante donnée aux régions, aux villes, aux quartiers, ce qui implique une possibilité d'initiative, d'autonomie de décisions, à tous les échelons. Surviennent un éclatement de l'espace de formation et l'assignation à la formation de nouvelles fonctions d'insertion professionnelle et sociale avec l'apparition de nouveaux 
publics engendrés par la crise socio-économique : jeunes auparavant exclus du système éducatif, jeunes sortis sans diplômes, migrants... Plusieurs rapports gouvernementaux pressent dans le sens d'un rapprochement entre entreprises et institutions de formation, dans un contexte de crise sociale et financière planétaire. Comme l'illustre ce texte proposé à la Commission européenne en avril 2009, proposant de donner une nouvelle légitimité aux entreprises en matière de formation et de professionnalisation :

17 «Le forum s'est penché sur la gouvernance aux niveaux national, régional et institutionnel comme une condition préalable à une collaboration efficace entre les universités et les entreprises. Au niveau national, des changements sont requis dans la législation, les modalités de financement et les structures incitatives, perçus comme peu favorables, voire défavorables dans certains cas, à la coopération entre les universités et les entreprises. Une telle coopération devrait faire partie de la stratégie globale de toutes les universités et s'inscrire dans leurs plans de développement et la définition de leurs objectifs. La collaboration avec l'industrie devrait être reconnue comme aussi importante pour l'avancement et la reconnaissance des mérites que des travaux universitaires tels que la publication. Les organismes intermédiaires ou les associations d'entreprises jouent un rôle important, car ils peuvent constituer une bonne interface entre les universités et les entreprises, notamment les PME. Par ailleurs, il existe plusieurs organismes ou agences de niveau européen, national ou régional qui soutiennent la coopération entre les universités et les entreprises. Ces structures peuvent s'avérer efficaces pour faire évoluer la situation. Une coopération efficace entre les universités et les entreprises est perçue comme un élément particulièrement important pour le développement régional. Le succès rencontré par de nombreuses régions aux États-Unis et en Europe est parti d'un partenariat triangulaire pour l'orientation stratégique et le financement, associant les universités, les entreprises et les pouvoirs publics. » (Commission européenne, 2009, p. 9-10).

\subsection{Le partenariat université-entreprise : une démarche peu valorisée, à risques}

Ces évolutions rentrent en confrontation avec ce que Barbier (2008) nomme le modèle production, transmission et application de savoirs, paradigme fondateur de l'université moderne dans sa référence humboltdienne, dominant dans l'enseignement supérieur et à la base de la gestion des activités des enseignants-chercheurs. Mais émerge, selon lui, un modèle plus récent, apparu au cours des années quatre-vingt du siècle dernier, celui qui « lie action, production de savoirs et construction des sujets humains» (p.113). Il est porté par des acteurs économiques, politiques et sociaux et par des personnalités caractérisées par leur mobilité entre espaces professionnels et espaces universitaires.

Plusieurs écueils guettent les universités pour s'ouvrir à un véritable partenariat. Il s'agit tout d'abord du faible intérêt porté encore aujourd'hui par cette institution aux questions de pédagogie universitaire, comme l'exprime Albéro, Linard et Robin (2008) pour la France, à la lumière de l'étude de quatre parcours d'universitaires dits « innovateurs » en matière d'enseignement et de formation : «La priorité accordée à la recherche dans la gestion des carrières ne fait que renforcer cette attitude. Une majorité d'universitaires continue d'ignorer la dimension pédagogique de l'enseignement. Limitant leur rôle à la transmission des contenus et des résultats de recherche, ils considèrent l'échec des étudiants comme un problème hors de leur 
responsabilité »(p.165-166). La persistance de ce déni académique du pédagogique avait déjà été repérée en son temps par Passeron (1991) à l'occasion d'une analyse de la "négation radicale d'une pédagogie des sciences». Elle est rejointe dans cette position par Côté et Allahar (2010). Ce problème se surajoute au manque d'ouverture de cette institution aux innovations pédagogiques. "Au plan institutionnel, on perçoit une ambiguïté du soutien des instances universitaires et les réticences de la reconnaissance académique quand l'innovation vient perturber ses règles, plus encore depuis les marges du système » (Albéro, Linard \& Robin, 2008, p. 194), un deuxième écueil qui ne facilite pas son ouverture, un maintien des références monodisciplinaires, notamment pour approcher les questions de professionnalisation des formations universitaires sur des positions corporatistes. C'est une position remise en question par Paul (2005) pour qui «Les nouveaux traits de la science se nomment complexité, paradoxe, hybridité, incomplétude, non-linéarité, hétérogénéité... C'est dans ce contexte que la réflexion transdisciplinaire peut trouver une bonne partie de ses sources. En particulier, la transdisciplinarité se distingue en ce qu'elle concerne ce qui est entre, au travers, au delà des disciplines » (p. 77).

Ce rapprochement université-entreprises n'est cependant pas dénué de risques car l'université est-elle un centre de formation comme un autre? Non, et elle a pu démontrer, tout au long de son histoire multiséculaire, qu'elle pouvait conserver sa véritable force qui est de maintenir des espaces propres de liberté et produire les ferments de l'esprit critique. Pour Charle et Verger (1994), c'est ce qui les distingue des autres organismes de formation. «Malgré toutes les transformations qu'ont connues les universités depuis le XIIIe siècle, cette fonction critique demeure sans doute le véritable fil rouge de cette aventure intellectuelle toujours menacée par les pouvoirs sociaux depuis sept siècles»(p.124). Pour Le Goff (1993), le risque majeur de la formation professionnelle y compris universitaire est de se retrouver à la remorque des entreprises, dans une logique utilitaire de court terme, les entreprises produisant ellesmêmes leurs critères d'excellence. Pour lui, nombre de responsables et d'éducateurs ont opéré une fuite en avant dans la liaison avec les entreprises qui aboutit à la remise en cause de la finalité éducative. L'entreprise et sa logique productive sont devenues une référence clé, les milieux universitaires n'échappant pas à ce culte de l'entreprise. En écho et en son temps, Ricoeur (1955) insistait sur la nécessaire distanciation et regard critique qu'impose le fait éducatif pour échapper à la dictature du courttermisme « La culture, c'est aussi ce qui désadapte l'homme, le tient prêt pour l'ouvert, pour le lointain, pour l'autre, pour le tout (...) L'éducation, au sens fort du mot, n'est peut-être que le juste mais difficile équilibre entre l'exigence d'objectivation, c'est-à dire l'adaptation, et l'exigence de réflexion et de désadaptation. C'est cet équilibre ténu qui tient l'homme debout» (p. 227-232). C'est en veillant à conserver cette ligne de crête entre nécessité de conserver ses spécificités historiques et identitaires, mais sans rigidité, et la nécessité de garder le contact avec les sociétés qui l'entourent que l'université pourra véritablement s'associer à d'autres partenaires, dans le cadre de tensions constitutives et productives.

21 Si les enjeux, défis, opportunités et risques pour l'université dans le cadre de la professionnalisation ont pu être approchés à travers ces premières pages, si les cadres des partenariats doivent être précisés pour éviter toute "fusion-confusion», quelles formes peuvent prendre ces partenariats visant professionnalisation? Comment repenser les dispositifs de formation universitaire pour qu'ils répondent pleinement à la demande sociale tout en garantissant l'autonomie des partenaires? Comment 
concevoir des dispositifs alternant apprentissages théoriques et pratiques, combinant des savoirs d'action et savoirs disciplinaires?

\section{L'alternance en pédagogie universitaire dans un cadre partenarial : une pluralité de formes et de finalités}

\subsection{L'expérience : notion centrale des dispositifs d'alternance}

Comme abordé précédemment, un nouveau type de pédagogie universitaire, en abordant les questions de professionnalisation, reconnaît explicitement que le travail est formateur et contribue à la construction de savoirs qui participent eux-mêmes de la construction des compétences professionnelles. Comme le précisait déjà Charlot dans les années quatre-vingt, ce phénomène traduit plus globalement une évolution du statut accordé à l'expérience de travail et de son rôle dans l'articulation entre formation et travail «La reconnaissance de la fonction formatrice du travail rappelle que l'école n'est pas la seule à former et qu'elle partage cette fonction avec d'autres instances éducatives parallèles" (Charlot, 1987, p. 124). Au sein des universités, la recherche s'est emparée de ces nouvelles perspectives en croisant notamment les théories de l'action, la sociologie compréhensive, l'interactionnisme social ou la perspective située. Elles présentent des démarches liées à l'analyse du travail, au récit autobiographique, à l'écriture réflexive, à la recherche-action ou encore à la didactique professionnelle (Dubar, 1998). Cette approche remet en question le système " humboldtien» de l'université appliquant le "modèle scolacentriste $»^{2}$ qu'il institue. Bourdoncle et Lessard (2002) relèvent que l'université a longtemps reposé sur ce modèle qui repose sur une triple conception :

- L'importance des diplômes pour assurer l'emploi et une entrée qualifiée dans la vie professionnelle, que seule l'institution peut délivrer;

- Celle de la place dominante accordée aux savoirs généraux et théoriques pour comprendre le monde et les choses, seuls capables de faire acquérir une autonomie de pensée, personnelle et professionnelle, de permettre à chacun de trouver dans des savoirs universels des réponses à des questions singulières ;

- Celle de l'enseignement et du mode pédagogique transmissif comme méthode pertinente d'appropriation des savoirs concernés, puisque fondée sur l'autorité de magisters reconnus pour leur capacité à inscrire les savoirs dans une histoire scientifique et sociale échappant aux aléas et à l'empirisme des contingences de situation, permettant ainsi à l'étudiant d'y reconnaître en l'élargissant sa propre histoire, et d'en tirer parti.

On voit comment, dans ce contexte, seul le savoir est reconnu, pas l'expérience qui n'est pas le savoir. L'activité professionnelle ne vaut que comme terrain d'application, ou lieu d'usage, d'ajustement des savoirs acquis en formation. Elle n'est qualifiée ni comme contenu, ni comme voie de formation recevable. Ou comme l'expriment Dubet et Martucelli (1998), l'unité de la personne aujourd'hui ne peut plus lui être donnée par un système homogène et des valeurs uniques, elle est celle de son activité réflexive et discursive. Pour Dubar et Tripier (1998), cette représentation "scolacentrée " proviendrait d'une vieille coupure entre ce qu'il nomme des savoirs sacrés et profanes. "Il existe une coupure interne entre les corps les plus nobles, issus des "arts libéraux ", composés d'intellectuels, formés dans les universités et les grandes écoles, 
et qui possèdent des connaissances désormais "scientifiques », et les corps les moins prestigieux, dérivés des "arts mécaniques" et constitués de travailleurs manuels formés par apprentissage auprès d'un patron, artisan ou commerçant qui appartient lui aussi à un " corps de métier » et qui ne possède pas ces connaissances-là. Cette dernière coupure sépare un sacré qui renvoie à un savoir théorique » (p. 33-34).

Comment cette alternance théorie-pratique est-elle valorisée aujourd'hui dans le cadre des formations universitaires? Plusieurs dispositifs sortent de notre propos car conçus hors de tout partenariat université-entreprise, comme les cursus de formation continue vécus au sein de l'université, à l'initiative des salariés (congés de formation, formations à visée promotionnelle ...). Nous relèverons principalement deux types de dispositifs qui rentreront dans ce cadre.

\subsection{L'analyse de pratique, forme spécifique d'alternance université- entreprise}

Les ateliers d'analyse de pratique en représentent la première forme. Ils sont pensés à partir de deux courants principaux : un courant d'orientation clinique et un courant d'orientation réflexive ou cognitive (Lagadec, 2010). Pratiqués depuis de nombreuses années dans le champ social, de l'éducation et de la santé dans de nombreux pays, ils représentent pour les employeurs et salariés un moyen de professionnalisation particulièrement recherché. Comme les présente Altet (2000), ils répondent aux modalités suivantes :

- C'est une démarche finalisée : elle vise la construction de l'identité professionnelle, la professionnalisation; c'est une démarche de formation ;

- C'est une démarche groupale : un membre du groupe expose sa pratique et, avec l'aide de ses pairs, tente de l'élucider, de lui donner du sens ;

- C'est une démarche accompagnée : le formateur est là pour donner des cadres de lecture, fournir des repères théoriques qui permettent de comprendre la situation. Il est aussi garant d'un cadre sécurisant pour le groupe ;

- C'est une démarche instrumentée par des savoirs, outils d'analyse : ces savoirs ont quatre dimensions : « une dimension instrumentale (formaliser la pratique), une dimension heuristique (ouvrir des pistes de réflexion), une dimension de problématisation (aider à poser les problèmes) et une dimension de changement (permettre la création de nouvelles représentations) » (p. 29);

- C'est une démarche et un lieu d'articulation pratique-théorie-pratique : elle part toujours de situations vécues apportées par le groupe. Les pairs questionnent, proposent des pistes d'analyse. L'accompagnateur apporte des référents théoriques, des réflexions argumentées. Mais ce qui est dominant, c'est la parole des membres du groupe. Ces dispositifs partenariaux valorisent l'expérience. Démarches qui s'apparentent au co-développement professionnel décrit par Payette et Champagne (2008) ou aux communautés de pratique décrites par Wenger (2005). Ils s'adressent à des professionnels adultes déjà aguerris et qui cherchent à progresser en s'inscrivant dans un trajet les poussant à cheminer de la pratique vers la théorie. Dans ce renversement du rapport au savoir, le formateur n'est plus le seul diffuseur de connaissances déjà écrites mais l'auxiliaire des praticiens à qui il fournit une aide pour formaliser le contenu de leur activité, confronter leurs concepts quotidiens aux concepts scientifiques afin d'en dégager la signification et le sens (Jobert, 1999). Ces dispositifs sont fréquemment conçus pour former des groupes de cadres, faciliter la 
cohésion de groupe et développer les compétences collectives. Cette voie de professionnalisation définie par Wittorski (2005) de « réflexion sur et par l'action » a pour particularité de transformer les pratiques en connaissances individuelles sur l'action, en connaissances partagées sur l'action et à produire de nouveaux savoirs pour l'action. Ils s'inscrivent dans une perspective d'apprentissage coopératif, d'une interdépendance positive des participants et s'opposent aux situations de type compétitifs et individualisants (Bourgeois, 1999). A titre individuel, ils sont identifiables également dans le coaching des dirigeants et des cadres supérieurs.

\subsection{L'alternance intégrative au sein des dispositifs diplômants universitaires}

L'alternance théorie-pratique dans un cadre partenarial peut également être identifiée dans des dispositifs s'adressant à des publics peu ou pas expérimentés, dans une visée certificative. En France, ils sont repérables au sein des licences professionnelles créées en 1999 ou au sein des masters professionnels issus du système européen LMD (licence, master, doctorat) qui s'institue au début des années deux mille. Ces dispositifs repensés valorisent l'expérience en faisant une place particulière aux pratiques de stage. Mais la présence d'un stage dans une formation universitaire n'est pas la garantie d'une formation alternée. Pour définir l'alternance véritable, de nombreux auteurs y ajoutent un qualificatif. Malglaive et Weber (1983) militent pour une alternance "réelle ou intégrative » ou " combinatoire » pour "jeter des ponts » entre mondes aux logiques opposées, Bourgeon (1979) comme « copulative " pour insister sur ses effets créateurs et productifs, mais aussi comme "associative" pour mettre en évidence le modèle ingénierique spécifique à conduire, Clavier (2001) comme « différentielle » pour insister sur le trajet et épreuves à dépasser dans un long processus interactif, parsemé d'embuches. Lerbet (1993), s'intéresse aux propriétés autonomisantes du sujet se construisant au cœur d'un tel dispositif. Tous s'accordent pour s'opposer à une alternance « juxtapositive » (Bourgeon, 1979), c'est-à-dire ne visant pas la synthèse des apports entre formation théorique et pratique et n'apportant qu'un statut mineur à celui de l'expérience accumulée en période de stage. Les définitions aujourd'hui de ce type d'alternance reprennent ses caractéristiques les plus représentatives. Ils insistent sur la présence des éléments suivants pour que le dispositif de formation soit reconnu comme « intégratif » :

- La reconnaissance de deux lieux, comme lieux de formation et d'apprentissage, pratique et théorique ;

- La progressivité de la formation conçue explicitement, que ce soit dans les contenus acquis en centre de formation que dans les expériences à vivre au sein de l'entreprise en situation réelle ;

-L'articulation organisée des apports de ces lieux, combinant apports théoriques et pratiques, se nourrissant l'un l'autre, temps et modalités d'apprentissage considérés comme structurant les parcours de formation ;

- L'alternance est organisée sous forme de partenariats entre l'entreprise et l'établissement de formation. Selon les cas, l'un ou l'autre des partenaires, plus rarement les deux, est maître d'œuvre. Elle met donc en jeu des acteurs appartenant à des univers professionnels dont les logiques diffèrent, logique de formation et logique de production ;

- Elle demande une concertation permanente, souvent polémique, qui devient un véritable travail de négociation continu et rigoureux des objectifs pédagogiques à atteindre, à partir 
de la connaissance et de la prise en compte des intérêts et des apports de chacun, concertation structurée autour d'espaces institués et associant les partenaires au début, en cours et à l'issue de la formation ;

- L'apprenant est au centre du dispositif et le principal acteur de ce processus intégrateur des savoirs. spécifique de formation pour organiser les liens entre "pratiques d'entreprise » et "savoirs universitaires ", comme le précisent Hahn, Besson, Collin, et Geay (2007), intégrant et combinant «des expériences au sein des cours, des études de cas interdisciplinaires, des «sas » de problématisation, des groupes d'analyse de retour d'expériences, des projets tutorés, un mémoire professionnel, une auto-évaluation des compétences... » (p. 24). Mais pour Lerbet-Sereni (2005), travailler dans ce paradigme, c'est d'emblée prendre une position scientifique non conforme. C'est par exemple, s'obliger à la multiréférentialité pour aborder son travail théorique. Car ce qui est en jeu dans l'alternance, c'est ce que l'on peut nommer "carrefour», "passage», «transdisciplinarité », " paradoxe », « problématisation », " production de sens », c'est l'attention focalisée sur tout ce qui à la fois relie et sépare, c'est-à-dire les dynamiques de la coupure-lien.

Trois auteurs, dans la deuxième partie de cette revue, abordent de manière centrale les problématiques liées au partenariat et aux dispositifs d'alternance. Tous trois conviennent que les expériences acquises au sein de partenariats et la pratique de dispositifs d'alternance s'accompagnent de la remise en question du paradigme encore dominant «scolacentré ».

Jean-François Savard complexifie son approche du partenariat en y introduisant des dimensions interculturelles. Il illustre à travers une expérience menée à partir de 2007, comment s'est nouée un partenariat assemblant deux institutions : l'École nationale d'administration publique (ENAP) mandatée par le gouvernement fédéral canadien et les gestionnaires du Conseil Cri de la santé et des services sociaux de la Baie-James (CCSSSBJ) associés à des gestionnaires de différentes communautés des Premières nations. Il s'agissait principalement pour l'ENAP de s'engager plus activement dans l'appui à la gouvernance en milieu autochtone en mettant en place des programmes de formation pour des gestionnaires des Premières nations déjà en poste. Dans une approche constructiviste, Jean-François Savard témoigne de la complexité d'un dispositif qui valorise une expérience par certains points exemplaires, et qui met en relief les écueils interculturels et interinstitutionnels qui se révèlent dans la conduite de projets d'un tel type, censés relier deux sociétés aussi différentes.

Hugues Pentecouteau replace la mise en œuvre des dispositifs alternants dans le cadre de l'évolution de l'université française. La mise en place des licences professionnelles marque selon lui un tournant, car supposant explicitement l'institution de partenariats et imposant l'alternance à travers un stage tutoré. Après avoir défini les différents types d'alternance à partir des principaux auteurs de référence, en tant que responsable du pilotage d'une licence professionnelle, il témoigne des défis à relever pour placer les questions posées par le travail et l'expérience qui en découle au cœur des apprentissages. Il précise les contours du dispositif mis en place, articulant des dimensions institutionnelles, comme les partenariats noués avec les employeurs et les initiatives prises en matière d'ingénierie pédagogique comme les rythmes choisis

Revue internationale de pédagogie de l'enseignement supérieur, 28(1) | 2012 
d'alternance, les spécificités de la progressivité de la formation, les innovations mises en place pour créer un mariage cognitif fort.

31 Catherine Guillaumin, quant à elle, illustre comment la remise en question du scolacentrisme est ancienne "au sein de l'école de Tours", sous l'impulsion de pionniers comme Georges Lerbet et Gaston Pineau, tous deux formés par l'école classique et qui comprirent très vite qu'il y avait d'autres voies pour apprendre et se former que celle de raisonner par accumulation. Ils seront perçus comme les véritables précurseurs en France des dispositifs d'alternance intégratrice. Catherine Guillaumin s'appuie sur l'expérience vécue avec ces pionniers au sein de deux masters professionnels alternants à parité d'estime pour en préciser les contours, les transformations subies à l'épreuve de l'expérience. Elle indique comment ont été prises en compte les évolutions de contexte pour s'adapter aux changements des milieux socio-économiques, qualifie la place offerte aux apprenants au cœur des dispositifs et illustre les caractéristiques des coopérations établies sur la base des réseaux et partenariats.

\section{Conclusion}

Pour clore cette introduction générale, et à travers les évolutions sociétales relevées, quelle place et quel rôle de l'université pouvons-nous entrevoir en matière de professionnalisation, dans un cadre partenarial ? Quelles perspectives et quels modèles d'université pouvons-nous promouvoir?

Bourdoncle et Lessard (2002) définissent trois types identitaires d'universitaires dans leur rapport à l'enseignement et la formation, reposant sur trois modèles de l'université :

- L'université libérale est celle du savoir désintéressé, des hommes libres, c'est-à-dire libérés du travail manuel. Elle défend une éducation générale qui apprend à faire face aux situations variées et considère la formation professionnelle comme une mauvaise chose car elle soumet l'homme à la logique utilitaire de travail ;

- Le modèle universitaire de recherche repose sur une communauté vouée à la recherche scientifique. Elle implique une forte symbiose entre l'enseignement et la recherche. La formation professionnelle n'y est reconnue que comme lieu d'investissement de la science et de son esprit ;

- Enfin, le modèle de l'université de service fait de l'université une institution au service du progrès social et savoir utile : ici, on postule que l'intelligence travaille mal dans le vide et cherche toujours à s'appuyer sur la rencontre de la théorie avec la pratique.

A l'issue de ce travail d'introduction à la problématique née de la rencontre entre pédagogie universitaire et partenariat université-entreprise, aucun de ces trois modèles ne semble correspondre aux positions émises par les différents contributeurs de cette revue thématique. Il conviendrait donc d'en inventer un quatrième, questionnant celui de l'université libérale, aujourd'hui dépassé, car remontant aux temps fondateurs de l'université médiévale. Mais aussi remettant en question le modèle universitaire de recherche, inspiré par Humboldt, trop scolacentré et limité aux sphères des États-nations, niant par là-même, les nouveaux espaces de la sociétémonde. Enfin, il nous faudrait nous méfier du modèle de l'université de service poussant l'université vers une démarche purement adéquationniste et la plaçant à la 
remorque d'un monde sur lequel le regard critique aurait du mal à s'affirmer, sous la pression du court-termisme.

A travers leurs positions, les contributeurs de cette revue défendent donc une position de l'université en ligne de crête, défendant l'établissement d'un quatrième modèle intégratif. Il s'agirait, alors, de concevoir des dispositifs de formation partenariaux, mais permettant à l'université de conserver ses caractéristiques principales: encourager l'éveil au regard critique sur les situations, développer les qualités de problématisation des apprenants, respectés comme acteurs de leur formation et non pas simplement soumis à leur condition d'élèves. Il conviendrait, par ailleurs, de laisser toute sa place à la recherche au sein des dispositifs de formation à la croisée des mondes université-entreprise, recherche au service d'une formation par production de savoirs (Pineau \& Marie-Michèle, 1983). Enfin, ce nouveau modèle en émergence serait le signe d'une conception plus ouverte de l'université, révélant un positionnement plus modeste, autorisant la surprise et la découverte et facilitant les interactions avec les mondes de la pratique. Elle y gagnerait en qualité de questionnement et en légitimité dans les espaces de l'espace-monde, tout en évitant les dérives soulignées par Lapointe (2011). Mais le plus grand défi qui se pose à la pédagogie universitaire est celui de la sensibilisation aux défis à relever en raison de l'évolution des missions des universités dans le monde d'aujourd'hui. Et faire évoluer les pratiques de formation partenariales en alternance, «c'est, on l'oublie trop souvent, former les enseignants » (Lerbet-Sereni, 2005, p. 334).

\section{BIBLIOGRAPHIE}

Albéro, B., Linard, M., \& Robin, J. Y. (2008). Petite fabrique de l'innovation à l'université. Paris : L'Harmattan.

Altet, M. (2000). L'analyse de pratiques : une démarche de formation professionnalisante? Recherche et formation, 35, 25-41.

Barbier, J. M., \& Galatanu, O. (dir.) (2004). Les savoirs d'action : une mise en mots des compétences? Paris : L'Harmattan.

Barbier, J.-M. (2008). Entretien : Formation et recherche. Ambiguïtés sémantiques et espaces d'action spécifique. Recherche et Formation, 59, 133-140.

Bernier, C. (2011). Introduction. Dans C. Bernier, L. Caron, N. Pépin \& M. Racine (dir.), Diversification des mains-d'œuvre, mobilisation des savoirs et formation (p. 1-19).Québec : Les presses de l'Université Laval.

Bézille, H., \& Courtois, B. (2006). Penser la relation expérience-formation. Lyon : Chronique sociale. Bourdoncle, R. (2000). Autour des mots : professionnalisation, formes et dispositifs. Recherche et Formation, 35, 117-132.

Bourdoncle, R., \& Lessard, C. (2002). Qu'est-ce qu'une formation professionnelle universitaire? Revue française de pédagogie, 139, 131-154. 
Bourgault, J., Charih, M., Maltais, D., \& Rouillard, L. (2003). Les rôles et les compétences des gestionnaires supérieurs du gouvernement du Québec pour l'avenir. Rapport de recherche. Québec: Gouvernement du Québec.

Bourgeois, E. (1999). Interactions sociales et performance cognitives. Dans P. Carré \& P. Caspar (dir.), Traité des sciences et des techniques de la formation (p. 289-303). Paris : Dunod.

Bourgeon, G. (1979). Sociopédagogie de l'alternance. Paris : UNMFREO.

Boutinet, J.P. (1999). Vie adulte et formation permanente : de la notion au concept. Dans P. Carré \& P. Caspar (dir.), Traité des sciences et des techniques de la formation (p. 169-187), Paris : Dunod.

Brémaud, L. (2010). Introduction générale : l'ingénierie de la formation, un monde en voie d'éclatement ou de recomposition? Dans L. Brémaud \& C. Guillaumin (dir.), L'archipel de l'ingénierie de la formation (p. 15-26). Rennes : Presses universitaires de Rennes.

Brémaud, L., \& Guillaumin, C. (dir.) (2010). L'archipel de l'ingénierie de la formation. Rennes : Presses universitaires de Rennes.

Charle, C., \& Verger, J. (1994). Histoire des universités. Paris : Que sais-je ? Presses universitaires de France.

Charlot, B. (1987). L'école en mutation. Paris : Fayot.

Clavier, L. (2001). Evaluer et former dans l'alternance. De la rupture aux interactions. Paris : L'Harmattan.

Clot, Y. (1995). Le travail sans l'homme ? Pour une psychologie des milieux de travail et de vie. Paris : La Découverte.

Commission européenne (2009). Un nouveau partenariat pour la modernisation des universités : le forum européen pour le dialogue université-entreprise. Communication de la Commission au parlement européen, au Conseil, au Comité économique et social européen et au Comité des Régions, Bruxelles, avril 2009.

Côté, J.E., \& Allahar, A. L. (2010). La tour de papier. L'université, mais à quel prix ? Montréal : Les Éditions Logiques.

Dadoy, M. (1990). De la qualification à la compétence. La sociologie du travail et la codification du social, 1, 232-241.

Debon, C. (2006). Parcours de la reconnaissance : le processus de validation des acquis de l'expérience. Dans N. Bézille \& B. Courtois (dir.), Penser la relation expérience-formation. (p. 188-205). Lyon : Chronique sociale.

Deledalle, G. (dir.) (1995). John Dewey. Paris : Presses universitaires de France.

Deleuze, G. (1989). Postface. Dans H. Melville (dir.), Bartleby, les îles enchantées (p. 186-197). Paris : Flammarion.

Demorgon, J. (2008). Avec Morin, du cosmos à l'humain, l'hypercomplexité comme représentation et comme volonté. Synergies Monde, 4, 97-145.

De Queiroz, J. M., \& Gruel, L. (1979). L'école et son dehors : réflexions sur le scolacentrisme des sociologies de la reproduction. Communication présentée aux journées d'études de la société française de sociologie, Bordeaux, juin 1979.

Dietrich, A., Gilbert, P., \& Pigeyre, F. (2010). Management des compétences : enjeux, modèles et perspectives. Paris : Dunod. 
Doray, P., \& Maroy, C. (1995). Les relations éducation-travail : quelques balises dans un océan conceptuel. Revue des sciences de l'éducation, 21(4), 661-668.

Dubar, C., \& Tripier, P. (1998). Sociologie des professions. Paris : Armand Colin.

Dubet, F., \& Martucelli, D. (1998). Dans quelle société vivons-nous? Paris : Stock.

Foucher, R. (2010). Gérer les talents et les compétences : principes, pratiques, instruments. Montréal : Éditions Nouvelles.

Freitag, M. (1998). Le naufrage de l'université et autres textes d'épistémologie politique. Montréal : Éditions Nota bene.

Geay, A. (2007). L'alternance comme processus de professionnalisation : implications didactiques. Education permanente, 172, 27-38.

Grosjean, G. (2005). La formation en alternance dans l'enseignement supérieur canadien. Dans C. Hahn, M. Besson, B. Collin, \& A. Geay (dir.), L'alternance dans l'enseignement supérieur, enjeux et perspectives (p. 223-240). Paris : L'Harmattan.

Hahn, C., Besson, M., Collin, B., \& Geay, A. (2005). Introduction : Pour une nouvelle approche de l'alternance dans l'enseignement supérieur. DansC. Hahn, M. Besson, B. Collin, \& A. Geay (dir.), L'alternance dans l'enseignement supérieur, enjeux et perspectives (p. 15-29). Paris : L'Harmattan.

Jobert, G. (1999). L'intelligence au travail. Dans P. Carré, \& C. Caspar (dir.), Traité des sciences et des techniques de la formation (p. 205-220). Paris : Dunod.

Labruffe, A. (2005). Management des compétences. Construire votre référentiel. Paris : AFNOR.

Lagadec, A.-M. (2010). L'analyse de pratiques comme moyen de développement des compétences : intérêts et limites. Dans L. Brémaud, \& C. Guillaumin (dir.), L'archipel de l'ingénierie de formation (p. 261-270). Rennes : Presses universitaires de Rennes.

Lapointe, P. A. (2011). Une démarche partenariale avec l'ARUC-Innovations, Travail et Emploi.Dans C. Bernier, L. Caron, N. Pépin \& M. Racine (dir.), Diversification des mains-d'œuvre, mobilisation des savoirs et formation (p. 197-217).Québec : Les presses de l'Université Laval.

Le Boterf, G. (2001). Construire les compétences individuelles et collectives. Paris : Éditions d'organisation.

Le Boterf, G. (2008). Repenser la compétence. Paris : Éditions d'organisation.

Le Gales, P. (2004). Gouvernance. Dans L. Boussaguet, S. Jacquot, \& P. Ravinet (dir.), Dictionnaire des politiques publiques (p. 256-257). Paris : Presses de Sciences Po.

Le Goff, J.-P. (1993). Le mythe de l'entreprise. Paris : Editions de la découverte.

Lerbet-Sereni, F. (2005). L'alternance à l'université : d'une ingénierie de formation à ses questionnements sous-jacents. Dans C. Hahn, M. Besson, B. Collin, \& A. Geay (dir.), L'alternance dans l'enseignement supérieur, enjeux et perspectives (p. 301-317). Paris : L'Harmattan.

Lerbet, G. (1993). Approche systémique et production de savoir. Paris : L'Harmattan.

Malglaive, G., \& Weber, A. (1983). École et entreprise : intérêt et limites de l'alternance en pédagogie. Revue française de pédagogie, 62, 51-64.

Martin, J. P. (2006). Travail, prendre en compte sa complexité croissante, vers la généralisation de la relation de service. Dans CAFOC de Nantes, Repenser la formation (p. 43-66). Lyon : Chronique sociale.

Mézirow, J. (2001). Penser son expérience, développer l'autoformation. Lyon : Chronique sociale. 
Parlier, M. (2009). Editorial. La construction des parcours professionnels. Education Permanente, 181, 5-10.

Passeron, J.-C. (1991). Le raisonnement sociologique. L'espace non poppérien du raisonnement naturel. Paris : Nathan, coll. Essais et Recherches.

Paul, P. (2005). Vers la transdisciplinarité ? Dans P. Paul \& G. Pineau (dir.), Transdisciplinarité et formation (p. 69-82). Paris : L'Harmattan.

Payette, A., \& Champagne, C. (2008). Le groupe de co-développement professionnel. Québec : Presses de l'Université du Québec.

Pineau, G., \& Marie-Michèle. (1983). Produire sa vie : autoformation et autobiographie. Paris : Edilig.

Ricoeur, P. (1955). Histoire et vérité. Paris : Seuil.

Squires, G. (1987). The curriculum beyond school. London : Hodder et Stoughton.

Voyer, B. (2011). Les professionnels en situation d'intermittence en emploi : enjeux pour la formation continue. Dans C. Bernier, L. Caron, N. Pépin \& M. Racine (dir.), Diversification des mainsd'œuvre, mobilisation des savoirs et formation (p. 85-108). Québec : Les presses de l'Université Laval.

Wenger, E. (2005). La théorie des communautés de pratiques : apprentissage, sens et identité. Québec : Les presses de l'Université Laval.

Wittorski, R. (2005). La contribution de l'analyse des pratiques à la professionnalisation des enseignants. Dans R. Wittorski (dir.), Formation, travail et professionnalisation (p. 23-49). Paris : L'Harmattan.

Wittorski, R. (2008). Professionnalisation et offre de formation en enseignement supérieur. Dans P. Hébrard \& C. Solar (dir.), Professionnalisation et formation des adultes: une perspective universitaire France-Québec (p. 47-70). Paris: L'Harmattan.

Zarifian, P. (1988). L'émergence du modèle de la compétence. Dans F. Stankiewicz (dir.), Les stratégies d'entreprise face aux ressources humaines. L'après Taylorisme (p. 75-90). Paris : Economica.

Zay, D. (1994). Définition de partenariat. Dans Dictionnaire encyclopédique de l'éducation et de la formation (p. 60-61). Paris : Nathan.

\section{NOTES}

1. Sources de ce paragraphe : Soubirou, A. (2004). La formation des élites en Europe. Eléments de cours. Paris : Institut d'études politiques.

2. Néologisme élaboré par De Queiroz et Gruel (1979) pour remettre en question une conception de la formation à dominante magistrale, programmatique et instituée, reposant sur ses aspects formels et sans réelles prises en compte des caractéristiques sociales des apprenants, ni des modes et conditions d'apprentissage des populations à former. 


\section{AUTEURS}

\section{LOÏC BRÉMAUD}

Maître de conférences, directeur de l'UFR (unité de formation et de recherche) sciences humaines.

Centre de recherche sur l'enseignement, les apprentissages et la didactique (CREAD), Université de Rennes 2, France.

loic.bremaud@univ-rennes2.fr

MICHEL BOISCLAIR

Chargé de projet, École nationale d'administration publique, Montréal, Québec, Canada. Doctorant à l'Institut de recherche sur les pratiques éducatives (IRPÉ)

Michel.boisclair@enap.ca 